\title{
Níveis de Interleucina-35 em Pacientes com Doença Arterial Coronariana Estável
}

\author{
Interleukin-35 Levels in Patients with Stable Coronary Artery Disease \\ Ersan Oflar, ${ }^{1}$ Mustafa Hakan Sahin, ${ }^{2}$ Bulent Demir, ${ }^{3}$ Abdulcelil Sait Ertugrul, ${ }^{1}$ Didem Melis Oztas, ${ }^{4}{ }^{\circledR 0}$ Metin Onur \\ Beyaz, ${ }^{5}$ Murat Ugurlucan, ${ }^{5}$ Fatma Nihan Turhan Caglar \\ Bakirkoy Dr Sadi Konuk Training and Research Hospital, Departamento de Cardiologia, ${ }^{1}$ Istambul - Turquia \\ Akdeniz Saglik Yasam Vakfi, Departamento de Cardiologia, ${ }^{2}$ Antália - Turquia \\ Turkish Hospital, Departamento de Cardiologia, ${ }^{3}$ Doha - Qatar \\ Bagcilar Training and Research Hospital, Departamento de Cirurgia Cardiovascular, ${ }^{4}$ Istambul - Turquia \\ Istanbul Medipol University Faculty of Medicine, Departamento de Cirurgia Cardiovascular, ${ }^{5}$ Istambul - Turquia
}

\section{Resumo}

Fundamento: Foi demonstrado que as subunidades de interleucina-35 (IL-35) estão fortemente expressas nas placas ateroscleróticas em humanos. Assim, considera-se que elas têm um papel na aterosclerose.

Objetivos: Neste estudo, os níveis de IL-35 foram comparados com o grupo controle em pacientes com doença arterial coronariana (DAC) estável, e a associação entre os níveis de IL-35 e o tipo, gravidade e extensão da lesão foram investigadas com o escore Gensini (GS) e o escore Syntax (SS) no grupo de pacientes

Métodos: Sessenta pacientes (18 mulheres e 42 homens) com DAC, diagnosticados por meio da angiografia coronária, que apresentaram dor no peito típica e teste de esforço não invasivo positivo, e 46 pacientes (18 mulheres e 28 homens) com luminograma normal, foram incluídos no estudo. Tanto o GS quanto o SS foram calculados para o grupo de pacientes, e esses valores foram comparados com os níveis de IL-35. Variáveis com distribuição não normal foram avaliadas com o teste $U$ de Mann-Whitney, enquanto os parâmetros com distribuição normal foram analisados com o teste $t$ de Student. A diferença entre as variáveis categóricas foi avaliada pelo teste de qui-quadrado ou de Fisher. Os valores de $\mathrm{p}<0,05$ foram considerados como estatisticamente sinificativos.

Resultados: Não foram observadas diferenças significativas entre pacientes e o grupo controle em termos de características demográficas e achados laboratoriais. Em comparação ao grupo controle, os níveis de IL-35 no grupo com DAC foram consideravalmente menores $(36,9 \pm 63,9 \mathrm{ng} / \mathrm{ml} \mathrm{vs} .33,2 \pm 13,2 \mathrm{ng} / \mathrm{ml}, \mathrm{p}<0,008)$. Embora não tenha sido estatisticamente significativo, os níveis de IL-35 foram maiores em pacientes com SS mais baixo do que nos com SS mais alto $(33,2 \pm 13,7$ vs. $31,8 \pm 8,9, p=0,51)$. Os valores de IL-35 em pacientes com GS alto foram significativamente mais baixos do que em pacientes com GS baixo ( $35 \pm 17,4$ vs. $30,7 \pm 8,6, p=0,043)$.

Conclusão: Demonstrou-se que os níveis de IL-35 podem ser um novo biomarcador para a DAC estável, e que a IL-35 está associada à extensão da DAC.

Palavras-chave: Aterosclerose; Doença da Artéria Coronariana; Interleucina-35; Pontuação de Propensão (Escore Gensini, Escore Syntax).

\footnotetext{
Abstract is considered to play a role in atherosclerosis. score (SS) in the patient group.

Correspondência: Fatma Nihan Turhan Caglar •

Bakirkoy Dr. Sadi Konuk Education and Research Hospital, Bakirkoy, Istambul, Turquia

E-mail: nhnturhan@gmail.com

Artigo recebido em 10/09/2020, revisado em 29/01/2021, aceito em 24/02/2021
}

Background: It has been shown that interleukin-35 (IL-35) subunits are strongly expressed in atherosclerotic plaques in humans. Therefore, it

Objectives: In this study, IL-35 levels were compared with the control group in patients with stable coronary artery disease (CAD), and the association between IL-35 levels and the lesion type, lesion severity and extension was investigated with the Gensini score (GS) and the Syntax

Methods: Sixty patients (18 female and 42 male) with CAD diagnosed by coronary angiography, who presented with typical chest pain and positive noninvasive cardiac stress test, and 46 patients (18 female and 28 male) with normal coronary lumenogram, were included in this study. Gensini and Syntax scores were calculated in the patient group, and these values were compared with IL-35 levels. Non-normally distributed

DOI: https://doi.org/10.36660/abc.20200945 
variables were analyzed by the Mann-Whitney $U$ test, whereas normally distributed parameters were assessed by Student's t-test. The difference between categorical variables were evaluated by the Chi-square or Fisher test. P-values $<0.05$ were considered as statistically significant.

Results: No significant differences were observed between patients and the control group in terms of demographic characteristics and laboratory findings. Compared to the control group, IL-35 levels of the CAD group were considerably lower $(36.9 \pm 63.9 \mathrm{ng} / \mathrm{ml} \mathrm{vs.} 33.2 \pm 13.2 \mathrm{ng} / \mathrm{ml}$, $p<0.008)$. Although not statistically significant, IL-35 levels were higher in patients with low SS than among those with high SS (33.2 \pm 13.7 vs. $31.8 \pm 8.9, p=0.51)$. The IL-35 values of the patients with high GS were significantly lower than in patients with low GS (35 \pm 17.4 vs. $30.7 \pm 8.6$, $p=0.043)$.

Conclusion: It has been shown that IL-35 levels can be a new biomarker for stable CAD, and IL-35 is associated with the extension of CAD.

Keywords: Coronary artery disease; Atherosclerosis; Interleukin-35; Gensini score; Syntax score.

Full texts in English - http://www.arquivosonline.com.br

\section{Introdução}

A doença arterial coronariana (DAC) é uma das principais causas de morte pelo mundo, embora a prevalência de mortalidade por DAC tenha recentemente apresentado queda na Europa e nos Estados Unidos. ${ }^{1,2}$ A DAC é uma disfunção progressiva e sistêmica causada pela aterosclerose. ${ }^{3}$

Embora a inflamação tenha papel importante no desenvolvimento da aterosclerose, o mecanismo que a causa não está claro até o momento. ${ }^{4}$ As citocinas anti-inflamatórias, como a interleucina-10 (IL-10), e o fator de crescimento transformante beta tipo 1 (TGF-1) são amplamento avaliados nos ensaios sobre aterosclerose..$^{5-11}$ Estudos publicados recentemente demonstraram que enquanto os níveis baixos de IL-10 e TGF-1 estão associados à progressão da aterosclerose e ao desenvolvimento da síndrome coronariana aguda, níveis altos de IL-10 e TGF-1 estão correlacionados ao bom prognóstico na DAC. ${ }^{5-11}$

A interleucina-35 (IL-35), uma citocina anfi-inflamatória recentemente definida, suprime a atividade das células CD4+T, induz a produção de células T regulatórias e reduz a progressão de doenças inflamatórias e autoimunes; por isso, tem um papel importante na aterosclerose. ${ }^{12-15}$

O objetivo deste estudo foi avaliar os níveis plasmáticos de IL-35 em pacientes com DAC estável e a associação entre IL-35 e a gravidade e extensão da DAC utilizando os escores Syntax (SS) e Gensini (GS).

\section{Métodos}

Este é um estudo transversal e observacional realizado em um centro de referência terciário. Cento e seis pacientes consecutivos, que foram submetidos à angiografia coronária diagnóstica no Bakırköy Dr. Sadi Konuk Research and Training Hospital - Clínica de Cardiologia, entre julho de 2018 e maio de 2019, foram incluídos no estudo. O grupo de pacientes consistia de 60 pacientes (42 homens e 18 mulheres) com DAC estável que apresentaram estenose arterial coronária epicárdica de mais de 50\% na angiografia coronária (AGC). O grupo controle consistia de 46 pacientes (28 homens e 18 mulheres) com dor no peito, mas AGC normal. Todos os pacientes tinham sido submetidos à avaliação objetiva da isquemia modificada, e todos tiveram resultados positivos em relação à isquemia. O número necessário de pacientes foi decidido com base em estudos prévios. $^{7}$ Os pacientes adequados para o estudo de acordo com os critérios de inclusão foram incluídos até atingirmos o número necessário de indivíduos entre julho de 2018 e maio de 2019.

O diagnóstico prévio de diabetes melitus (DM), o uso de remédios para diabetes ou o nível de glicose em jejum de $126 \mathrm{mg} / \mathrm{dl}$ em dois momentos, em pacientes previamente não tratados, foram necessários para diagnosticar a DM. A hipertensão (HT) foi definida com base no uso prévio de medicações para hipertensão, pressão sistólica maior que $140 \mathrm{mmHg}$, ou pressão diastólica maior que $90 \mathrm{mmHg}$ em pelo menos dois momentos separados. A taxa de filtração glomerular (TFG) foi estimada utilizando a equação da Modificação de Dieta em Doença Renal (MDRD) na internação. O Índice de Massa Corporal (IMC) foi calculado de acordo com os critérios da Organização Mundial da Saúde (OMS). A hiperlipidemia (HL) foi definida com base no uso prévio de antilipêmicos nos seis meses anteriores, ou altos níveis de lipídios séricos medidos após 8 horas de jejum [lipoproteína de baixa densidade (LDL) $>160 \mathrm{mg} / \mathrm{dl}$, colesterol total (CT) > $240 \mathrm{mg} / \mathrm{dl}$, ou triglicérides (TG) >160 mg/dl)].

Todos os pacientes assinaram um termo de consentimento, e este estudo está de acordo com a Declaração de Helsinki. Nossa pesquisa foi aprovada pelo Conselho de Ética Institucional.

Pacientes com DAC diagnosticada, TFG estimada (TFGe) $<60 \mathrm{ml} / \mathrm{min}$, aqueles com doença valvar, indivíduos com pressão arterial sistólica maior que $140 \mathrm{mmHG}$ e pressão arterial diastólica maior que $90 \mathrm{mmHg}$ apesar do tratamento, insuficiência cardíaca, insuficiência renal, infecção aguda/crônica, febre, dores musculares, dores de cabeça, indivíduos em uso de antibióticos, indivíduos com doença imunoproliferativa, doença reumática, câncer, osteoporose e aqueles com mais de 75 anos foram excluídos.

\section{Avaliação objetiva da isquemia}

Todos os pacientes tinham sido submetidos a um teste de esforço não-invasivo para a avaliação da isquemia. Na maior parte dos casos, o protocolo de Bruce modificado foi realizado. Uma depressão de segmento ST de pelo menos $1 \mathrm{~mm}$, horizontal ou decrescente, em derivações $\geq 2$ após 60*80 milissegundos desde o ponto J durante o exercício, foi considerada anormal. O escore de Duke foi usado para a estratificação do risco. ${ }^{11}$ Uma AGC foi realizada em pacientes que tinham escore de Duke médio ou alto. Uma cintilografia do miocárdio perfusão (CMP) foi realizada para avaliar a isquemia em pacientes internados com bloqueio 
atrioventricular de primeiro grau ou depressão de ST $\geq 1 \mathrm{~mm}$ no eletrocardiograma de repouso (ECG), teste de esforço não diagnóstico, ou com pouca capacidade de esforço. A AGC foi realizada em pacientes que apresentavam nível de isquemia médio ou alto na CMP.

\section{Medidas de biomarcadores}

Todos os dados laboratoriais dos pacientes, como troponina cardíaca $\mathrm{T}(\mathrm{TnT})$, creatinina, contagem de leucócitos, proteína $\mathrm{C}$ reativa de alta sensibilidade (PCR-as) etc., foram documentados.

Amostras sanguíneas para IL-35 foram coletadas no laboratório de cateterização antes da angiografia coronária em todos os participantes. As amostras foram obtidas por punção venosa com uso de EDTA (ácido etilenodiaminotetracético), em tubos sem aditivos, e imediatamente centrifugadas a 4.000 rpm por 10 minutos. As amostras foram coletadas após a centrifugação e armazenadas a -800 $\mathrm{C}$ até a análise (não mais do que 6 meses).

As amostras foram descongeladas somente uma vez. A técnica do sanduíche, duplo anticorpo à base de biotina, utilizando o kit ELISA, foi usada para a análise de IL-35 (interleucina 35 humana: Yehua Biological Technology; Cat No:YHB1739Hu). A IL-35 foi adicionada a poços prérevestidos com o anticorpo monoclonal IL-35, e, depois, incubada. Após a incubação, os anticorpos anti-IL-35 identificados com biotina foram adicionados à unidade com estreptavidina-HRP para formar o complexo imune. Após a lavagem, as enzimas não ligadas foram removidas e, depois, adicionadas aos substratos A e B. A solução tornou-se azul e transformou-se em amarelo com o efeito do ácido. Os tons da solução e a concentração de IL-35 estiveram positivamente correlacionados. Os resultados foram expressos em ng/ml. Os coeficientes de variação $(\mathrm{CV})$ intra e entre ensaios da análise foram de $<10 \%$ e $<12 \%$, respectivamente.

\section{Angiografia coronária}

Todos os procedimentos de AGC foram realizados com o técnica de Judkins, por meio de um procedimento de cineangiografia (Axiom Artis, Siemens, Alemanha). Todas as angiografias foram registradas em compact discs (CDs), no formato DICOM, e visualmente examinadas por dois cardiologistas intervencionistas experientes e cegos para o estudo. A gravidade e extensão da DAC foram avaliadas de acordo com os SS e GS. O nível de estreitamento do lúmen, a concentricidade e a excentricidade foram avaliados. De acordo com o GS, 1 ponto é dado para 1-25\% de estenose; 2 pontos, de $26-50 \%$; 4 pontos, de $51-75 \%$; 8 pontos, de 76-90\%; 16 pontos, de 91-99\%; e 32 pontos para 100\%. Depois, o número de pontos para cada lesão é multiplicado pelo coeficiente considerado para cada segmento vascular principal, de acordo com a significância funcional do vaso (artéria coronária principal esquerda x 5; segmento proximal da artéria coronária descendente anterior esquerda x 2,5; segmento proximal da artéria circunflexa x 2,5; segmento médio da artéria coronária descendente anterior esquerda $\times 1,5$; artéria coronária direita, segmento distal da artéria coronária descendente anterior esquerda, artéria posterolateral e artéria obtusa marginal $\times 1$; e outros $\times 0,5$ ), e a soma de todos os pontos constitui a pontuação total. ${ }^{16}$ O escore foi realizado com dois observadores e o valor médio. GS $<20$ foi considerado como DAC leve (grupo 1), e GS $\geq 20$ foi considerado como DAC grave (grupo 2). A SS correspondendo à complexidade da lesão foi medida pelas características da árvore coronária, assim como a localização da lesão e suas especificidades. ${ }^{17} \mathrm{O}$ escore é medido por meio de uma calculadora virtual de acesso livre (www.syntaxscore. com). A pontuação foi realizada e a média foi calculada por dois observadores, cegos aos grupos do estudo.

\section{Análise estatística}

A análise estatística foi realizada com o software SPSS, versão 16. A distribuição normal das variáveis contínuas foi analisada por métodos visuais (historama) e analíticos (Kolmogorov-Smirnov). As variáveis contínuas com distribuição normal foram demonstradas como média \pm desvio padrão (DP). Variáveis contínuas com distribuição não normal foram demonstradas como mediana e intervalo interquartil. As variáveis de distribuição não normal foram avaliadas com o teste $U$ de Mann-Whitney, enquanto os parâmetros de distribuição normal foram analisados pelo test t de Student não pareado. As diferenças entre as variáveis categóricas foram avaliadas pelo teste de qui-quadrado ou de Fisher. A associação entre variáveis de distribuição não normal foi analisada pelo teste de Spearman; por outro lado, o teste de Pearson foi usado para a correlação entre as variáveis de distribuição normal. O teste de qui-quadrado foi usado para a sensibilidade, especificidade, valores preditivos negativos e positivos. A análise de regressão logística foi realizada para demonstrar a IL-35 como um fator de risco independente da DAC entre os fatores de risco tradicionais. A efetividade e a compatibilidade do modelo criado foram verificadas com o teste de Hosmer-Lemeshow. Valores de $p<0,05$ foram considerados como estatisticamente significativos.

\section{Resultados}

Os aspectos demográficos dos grupos de pacientes e controle estão demonstados na Tabela 1. Da mesma forma, a idade média do grupo de pacientes foi maior do que no grupo controle. Gênero, hábito de fumar, DM, HT, HL e IMC foram semelhantes entre os grupos (Tabela 1). Os achados laboratoriais dos grupos estão demonstrados na Tabela 2. Os níveis de IL-35 foram significativamente menores no grupo de pacientes em relação ao grupo controle $(p=0,008)$ (Figura 1). Além disso, enquanto a contagem de leucócitos, níveis de colesterol total e lipoproteína de baixa densidade (LDL) estavam maiores no grupo de pacientes, a contagem de trombócitos foi mais baixa no grupo de pacientes em comparação ao grupo controle (Tabela 2). A Tabela 3 ilustra os níveis de IL-35 de acordo com os principais aspectos demográficos e laboratoriais no grupo de pacientes. Da mesma forma, os níveis de IL-35 foram significativamente mais baixos em indivíduos com diabetes no grupo de pacientes $(p=0,042)$. Analisamos mais profundamente os níveis de IL-35 em ambos os grupos, em pacientes com e sem diabetes, para demonstrar se os níveis baixos de IL-35 em pacientes com DAC estavam relacionados à diabetes ou não (Tabela 4). Os níveis de IL-35 não estavam associados à presença de diabetes $(p=0,18)$. Embora os níveis de IL-35 tenham sido 


\begin{tabular}{|c|c|c|c|}
\hline & Paciente, n(\%) & Controle, $n(\%)$ & Valor de $p$ \\
\hline Total & $60(100)$ & $46(100)$ & \\
\hline Idade (média $\pm D P$ ) & $59 \pm 9,1$ & $54,5(8,9)$ & $0,013^{*}$ \\
\hline \multicolumn{4}{|l|}{ Sexo } \\
\hline Masculino & $42(70)$ & $28(60,9)$ & 0,32 \\
\hline Feminino & $18(30)$ & $18(39,1)$ & \\
\hline Fumar & $38(63,3)$ & $12(26,1)$ & $<0,001$ \\
\hline Diabetes & $22(36,7)$ & $10(21,7)$ & 0,09 \\
\hline Hipertensão & $38(63,3)$ & $21(45,7)$ & 0,07 \\
\hline Hiperlipidemia & $16(26,7)$ & $9(19,6)$ & 0,008 \\
\hline IMC (média $\pm D P)$ & $26,3 \pm 4,4$ & $28.2 \pm 3,8$ & $0,07^{*}$ \\
\hline
\end{tabular}

Teste de qui-quadrado, *teste t de Student; IMC: índice de massa corporal.

Tabela 2 - Achados laboratoriais nos grupos de pacientes e controles

\begin{tabular}{|c|c|c|c|}
\hline & Grupo de pacientes & Grupo controle & Valor de $p$ \\
\hline IL-35 (pg/ml) & $\begin{array}{c}\text { Mediana: } 33,2 \\
\text { Intervalo: } 23,39-172,6\end{array}$ & $\begin{array}{c}\text { Mediana: } 36,9 \\
\text { Intervalo: } 23,39-238,1\end{array}$ & 0,008 \\
\hline Creatinina (mg/dl) & $\begin{array}{c}\text { Mediana: } 0,8 \\
\text { Intervalo: } 0,35-1,1\end{array}$ & $\begin{array}{c}\text { Mediana: } 0,8 \\
\text { Intervalo: } 0,42-1,2\end{array}$ & 0,43 \\
\hline Colesterol LDL (mg/dl)(média+DP) & $149 \pm 39,3$ & $116,5 \pm 35,9$ & $<0,001^{*}$ \\
\hline Colesterol total (mg/dl) (média+DP) & $229,5 \pm 47,1$ & $190,5 \pm 46,3$ & $<0,001^{*}$ \\
\hline Colesterol HDL (mg/dl) & $\begin{array}{c}\text { Mediana: } 44 \\
\text { Intervalo: } 25-72\end{array}$ & $\begin{array}{c}\text { Mediana: } 45 \\
\text { Intervalo: } 27-72\end{array}$ & 0,98 \\
\hline Triglicérides (mg/dl) (média+DP) & $150,5 \pm 81$ & $115,5 \pm 83,2$ & $0,19^{*}$ \\
\hline $\begin{array}{l}\text { Contagem de leucócitos }\left(10^{3} / \mathrm{mm}^{3}\right) \\
\text { (média+DP) }\end{array}$ & $8250 \pm 2670$ & $6550 \pm 2030$ & $0,006^{*}$ \\
\hline Hematócrito (\%) & $\begin{array}{c}\text { Mediana: } 40,8 \\
\text { Intervalo: } 29,7-52,1\end{array}$ & $\begin{array}{c}\text { Mediana: } 40,9 \\
\text { Intervalo: } 28,6-50,2\end{array}$ & 0,93 \\
\hline $\begin{array}{l}\text { Hemoglobina }(g / d l) \\
\text { (média+DP) }\end{array}$ & $13,9 \pm 1,7$ & $13,4 \pm 1,8$ & $0,37^{*}$ \\
\hline \multirow[t]{2}{*}{$\begin{array}{l}\text { Contagem de trombócitos }\left(10^{3} / \mathrm{mm}^{3}\right) \\
\text { (média+DP) }\end{array}$} & $248 \pm 104$ & $270 \pm 64,7$ & $0,015^{*}$ \\
\hline & & Mediana: 2,6 & 0,09 \\
\hline PCR-as (mg/L) & $\begin{array}{c}\text { Mediana: } 4,1 \\
\text { Intervalo: } 0,32-18,2\end{array}$ & Intervalo: $0,2-17,8$ & \\
\hline $\mathrm{HbA} 1 \mathrm{C}(\%)$ & $\begin{array}{c}\text { Mediana: } 5,9 \\
\text { Intervalo: } 4,0-13,2\end{array}$ & $\begin{array}{c}\text { Mediana: } 5,8 \\
\text { Intervalo: } 4,5-7,6\end{array}$ & 0,45 \\
\hline
\end{tabular}

IL-35: Interleucina-35; LDL: lipoproteina de baixa densidade; HDL: lipoproteina de alta densidade; PCR-as: proteína C reativa de alta sensibilidade; HbA1C: Hemoglobina A1C. Teste U de Mann-Whitney, *teste $t$ de Student 


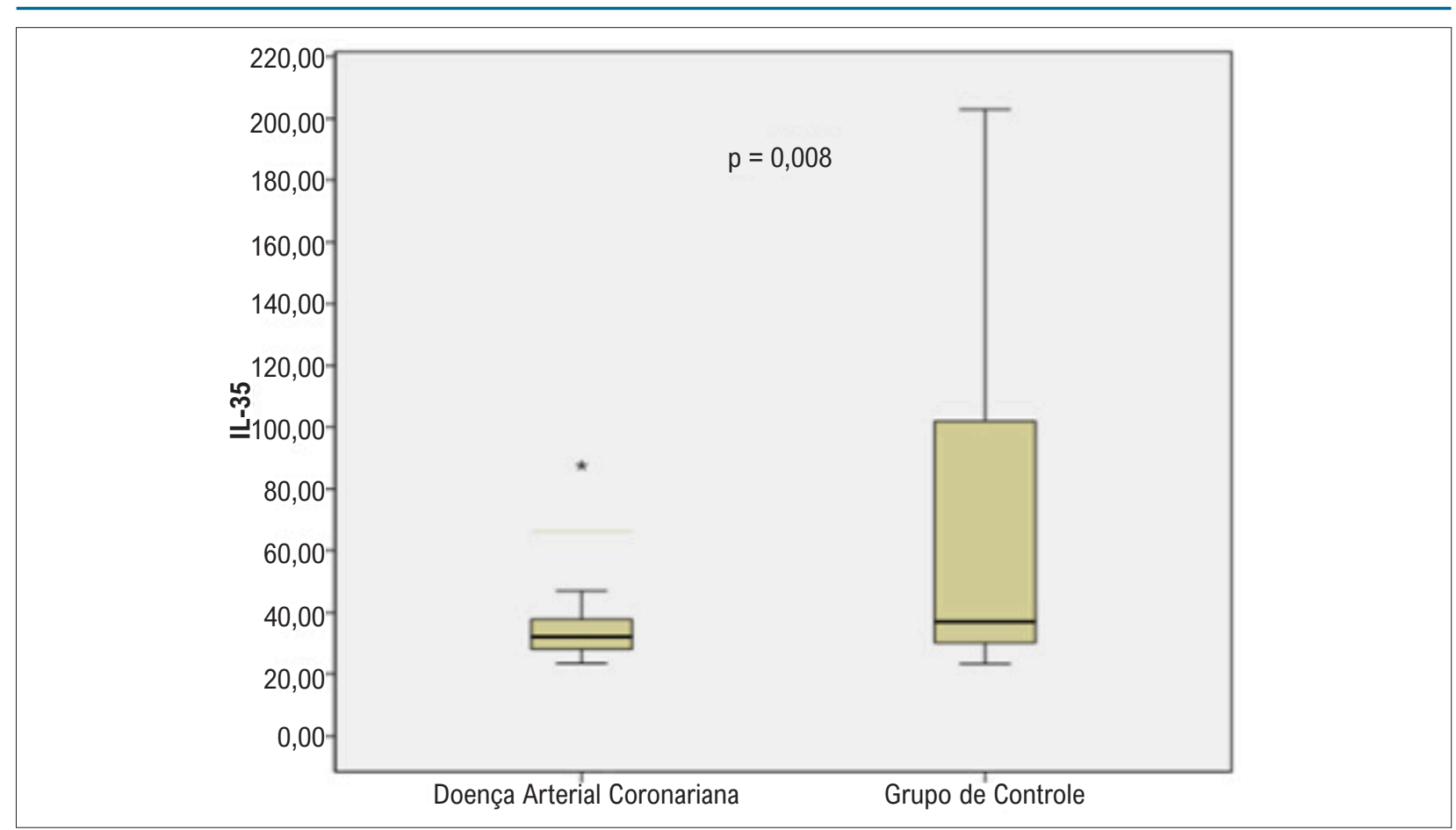

Figura 1 - Niveis de IL-35 nos grupos de pacientes e controles (Box-plot).

semelhantes entre os pacientes com DAC com SS baixo $(<22)$ e alto $(\geq 22)$, foram menores em pacientes com DAC com GS alto $(\geq 20)$ do que entre aqueles com GS baixo $(<20)(p=0,51$ e $p=0,043$, respectivamente) (Tabela 5). A análise de correlação entre IL-35 e outros parâmetros está demonstrada na Tabela 6 . Os níveis de IL-35 tinham uma correlação negativa leve com o SS e com os níveis de colesterol total $(p=0,036)$ (Figura 2) (Tabela 6). Por fim, na análise de regressão logística que incluía IL-35, HM, gênero, idade, HT, DM tipo 2 e hábito de fumar, somente a DM tipo $2(p=0,049, R R=3,44, C l: 1,004-11,8)$, o hábito de fumar ( $p<0,001, R R=11,27, \mathrm{IC}: 3,45-36,83) \mathrm{e}$ os níveis de IL-35 ( $p=0,017, R R=1,02, G A: 1,005-1,053)$ tiveram um efeito independente na presença da DAC (Tabela 7). O ponto de corte para os níveis de IL-35 para detectar a DAC foi a avaliado pela análise ROC (Figura 3) (Tabela 8).

\section{Discussão}

Sabe-se que a aterosclerose é multifatorial e está muito relacionada à inflamação. ${ }^{4} \mathrm{O}$ equilíbrio entre as citocinas proinflamatórias e anti-inflamatórias está associado à estabilidade das placas e à progressão da aterosclerose. ${ }^{4,5}$ Baixos níveis de IL-35 são indicativos tanto da resposta anti-inflamatória insuficiente quanto do nível da inflamação na DAC. ${ }^{18,19}$ Normalmente, a IL-35 só é induzida com inflamação, e depois detectada no sangue periférico. ${ }^{19} \mathrm{Em}$ um estudo conduzido com ratos, a IL-35 não foi detectada no perfil tecidual de sujeitos saudáveis; porém, estava aumentada em amostras teciduais de ratos com resposta inflamatória formada externamente. ${ }^{20}$

A expressão de IL-35 e os níveis sanguíneos foram avaliados por muitos pesquisadores em cenários clínicos diferentes. ${ }^{20-23}$ Kempe et al., ${ }^{21}$ demonstraram forte expressão de IL-35 nas células endoteliais, células do músculo liso vascular e macrófagos de pacientes sintomáticos com placas nas carótidas, enquanto não poderiam detectar a expressão de IL-35 na camada íntima da carótida saudável. ${ }^{21}$

Por outro lado, há uma relação linear entre a gravidade da inflamação e a baixa IL-35 na DAC. ${ }^{21}$ A resposta anti-inflamatória inadequada na aterosclerose leva à resposta inflamatória excessiva na placa. ${ }^{21} \mathrm{Em}$ outras palavras, quanto mais baixos os níveis de IL-35, mais alta a carga aterosclerótica nas artérias coronárias. $^{22}$ Da mesma forma, Yanmei et al. demonstraram uma correlação entre níveis decrescentes de IL-35 e a gravidade da doença inflamatória intestinal. ${ }^{22}$ Esses dados sugerem que, primeiro, a IL-35 aumenta de forma secundária à inflamação, e depois diminui conforme a inflamação se torna mais grave. ${ }^{22}$

No grupo de pacientes, os níveis de IL-35 foram significativamente mais baixos em pacientes com GS alto em comparação àqueles com GS baixo $(35 \pm 17,4$ vs. $30,7 \pm 8,6$, $p=0,043)$. Por outro lado, os níveis de IL-35 foram semelhantes entre pacientes com CAD com SS baixo e alto $(31,8 \pm 8,9$ vs. $33,2 \pm 13,7, p=0,51)$; porém, em nossa opinião, o número de pacientes com SS alto foi muito pequeno para uma análise significativa. Um motivo para incluirmos poucos pacientes com SS alto pode ser o fato de termos consideraedo somente pacientes com DAC estável, e não indivíduos com síndrome coronária aguda nem aqueles com histórico de DAC. Nossos achados são únicos porque, até onde sabemos, este estudo é o primeiro que revelou uma correlação entre a gravidade da DAC e os níveis de IL-35. Recentemente, Lin et al., ${ }^{18}$ demonstraram níveis mais baixos de IL-35 em pacientes com DAC estável e infarto agudo do miocárdio (IAM) em comparação ao grupo controle; porém, não avaliaram o efeito da extensão e gravidade da DAC..$^{18}$ Os níveis mais baixos de IL-35 estão no grupo IAM do estudo de Lin. ${ }^{18}$ Assim, especulou-se que a IL-35 pode ser usada como preditor do desfecho clínico para aterosclerose. ${ }^{19}$ 
Tabela 3 - Níveis de IL-35 de acordo com os principais aspectos demográficos e laboratoriais no grupo de pacientes

\begin{tabular}{|c|c|c|}
\hline & Mediana/Intervalo Interquartil & Valor de $p$ \\
\hline Sexo & & 0,55 \\
\hline Masculino & $\begin{array}{c}\text { Mediana: } 34,7 \\
\text { Intervalo: } 23,39-103,9\end{array}$ & \\
\hline Feminino & $\begin{array}{c}\text { Mediana: } 34,3 \\
\text { Intervalo: } 26,5-172,6\end{array}$ & \\
\hline Idade & & 0,12 \\
\hline$<50$ & $\begin{array}{c}\text { Mediana: } 38,1 \\
\text { Intervalo: } 27,2-172,6\end{array}$ & \\
\hline$\geq 50$ & $\begin{array}{c}\text { Mediana: } 34,7 \\
\text { Intervalo: } 23,9-133,3\end{array}$ & \\
\hline $\begin{array}{l}\text { Hipertensão } \\
(+)\end{array}$ & $\begin{array}{c}\text { Mediana: } 34 \\
\text { Intervalo: } 23,39-172,6\end{array}$ & 0,11 \\
\hline$(-)$ & $\begin{array}{c}\text { Mediana: } 35,8 \\
\text { Intervalo: } 25,4-80,61\end{array}$ & \\
\hline $\begin{array}{l}\text { Diabetes } \\
(+)\end{array}$ & $\begin{array}{c}\text { Mediana: } 30,4 \\
\text { Intervalo: } 23,9-172,6\end{array}$ & 0,042 \\
\hline$(-)$ & $\begin{array}{c}\text { Mediana: } 35,0 \\
\text { Intervalo: } 24,16-133,3\end{array}$ & \\
\hline $\begin{array}{l}\text { Fumar } \\
(+)\end{array}$ & $\begin{array}{c}\text { Mediana: } 35,0 \\
\text { Intervalo: } 27,11-133,3\end{array}$ & 0,5 \\
\hline$(-)$ & $\begin{array}{c}\text { Mediana: } 34,1 \\
\text { Intervalo: } 23,39-172,6\end{array}$ & \\
\hline $\begin{array}{l}\text { Hiperlipidemia } \\
(+)\end{array}$ & $\begin{array}{c}\text { Mediana: } 34,6 \\
\text { Intervalo: } 25,4-133,3\end{array}$ & 0,56 \\
\hline$(-)$ & $\begin{array}{c}\text { Mediana: } 34,1 \\
\text { Intervalo: } 23,39-172,6\end{array}$ & \\
\hline IMC & & 0,71 \\
\hline $\mathrm{IMC}<25$ & $\begin{array}{c}\text { Mediana: } 34,8 \\
\text { Intervalo: } 24,16-56,12\end{array}$ & \\
\hline $\mathrm{IMC} \geq 25$ & $\begin{array}{c}\text { Mediana: } 34,2 \\
\text { Intervalo: } 23,9-172,6\end{array}$ & \\
\hline IMC & & 0,13 \\
\hline $\mathrm{IMC}<30$ & $\begin{array}{c}\text { Mediana: } 35 \\
\text { Intervalo: } 25,3-172,6\end{array}$ & \\
\hline $\mathrm{IMC} \geq 30$ & $\begin{array}{c}\text { Mediana: } 30,7 \\
\text { Intervalo: } 23,9-87,6\end{array}$ & \\
\hline PCR-as & & 0,61 \\
\hline$<5 \mathrm{mg} / \mathrm{L}$ & $\begin{array}{c}\text { Mediana: } 34,6 \\
\text { Intervalo: } 24,16-87,51\end{array}$ & \\
\hline$\geq 5 \mathrm{mg} / \mathrm{L}$ & $\begin{array}{c}\text { Mediana: } 33,4 \\
\text { Intervalo: } 23,9-172,6\end{array}$ & \\
\hline
\end{tabular}

IMC: índice de massa corporal; $P C R$-as: proteina $C$ reativa de alta sensibilidade.

Tabela 4 - Níveis de IL-35 de acordo com o histórico de diabetes em ambos os grupos

\begin{tabular}{ccccc}
\hline & Variáveis & DAC(+) & DAC(-) & p \\
\hline DM(-) & IL-35 & Mediana: 34,4 & Mediana: 40,6 & 0,021 \\
\hline DM(+) & IL-35 & Mediana: 29,6 & Intervalo: 24,59-238,1 & Intervalo: 23,39-172,6 \\
\hline
\end{tabular}

DAC: doença arterial coronariana 


\section{Artigo Original}

Tabela 5 - Níveis de IL-35 no grupo de pacientes de acordo com os escores Gensini e Syntax

\begin{tabular}{lcccc}
\hline & & Número de pacientes (\%) & IL-35(média \pm DP) & Valor de $\mathbf{p}$ \\
\hline Escore Gensin & $<20$ & $23(38,3)$ & $35 \pm 17,4$ & $0,043^{*}$ \\
\hline Escore Syntax & $\geq 20$ & $37(61,7)$ & $30,7 \pm 8,6$ & \\
\hline & $<22$ & $52(86,7)$ & $33,2 \pm 13,7$ & $0,51^{*}$ \\
\hline
\end{tabular}

* Teste $t$ de Student, $¥$ Teste U de Mann-Whitney.

Tabela 6 - Análise de correlação entre a IL-35 e outros parâmetros

\begin{tabular}{lccccccc}
\hline Variáveis & & Rho & Valor de $\mathbf{p}$ & & Variáveis & Rho & Valor de $\mathbf{p}$ \\
\hline IL-35 & Colesterol total & $-0,204$ & 0,036 & IL-35 & Escore Gensini & $-0,208$ & 0,11 \\
\hline IL-35 & Leucócitos & 0,12 & 0,2 & IL-35 & Escore Syntax & $-0,293$ & 0,023 \\
\hline IL-35 & PCR-as & $-0,03$ & 0,75 & & & & \\
\hline
\end{tabular}

PCR-as: proteína C reativa de alta sensibilidade; Rho: coeficiente de correlação de teste de Spearman ou $\rho$ de Spearman.

Tabela 7 - Análise de regressão logística dos principais parâmetros para previsão da DAC

\begin{tabular}{lccc}
\hline Parâmetros & RR & IC95\% & Valor de $\mathbf{p}$ \\
\hline Hiperlipidemia & 1,22 & $0,34-4,34$ & 0,75 \\
\hline Sexo & 0,9 & $0,27-2,98$ & 0,87 \\
\hline Idade & 0,96 & $0,91-1,02$ & 0,23 \\
\hline Hipertension & 17 & $0,56-5,15$ & 0,34 \\
\hline Diabetes & 3,44 & $1,004-11,8$ & 0,049 \\
\hline Fumar & 11,27 & $3,45-36,83$ & $<0,001$ \\
\hline IL-35 & 1,02 & $1,005-1,053$ & 0,017 \\
\hline
\end{tabular}

RR: razão de risco; IC: intervalo de confiança; DAC: doença arterial coronariana.

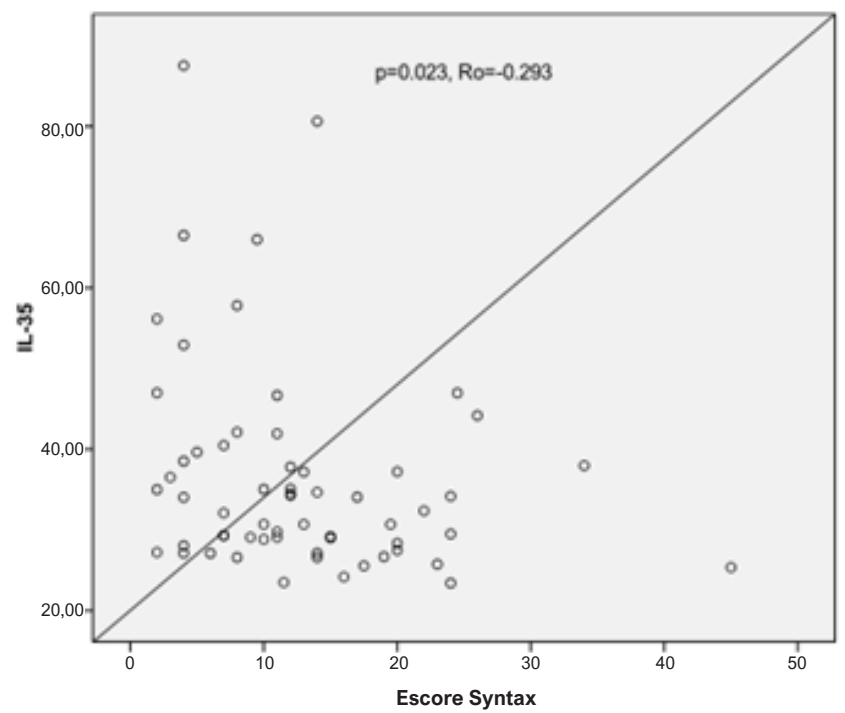

Figura 2 - Associação entre níveis de IL-35 e o escore Syntax. 


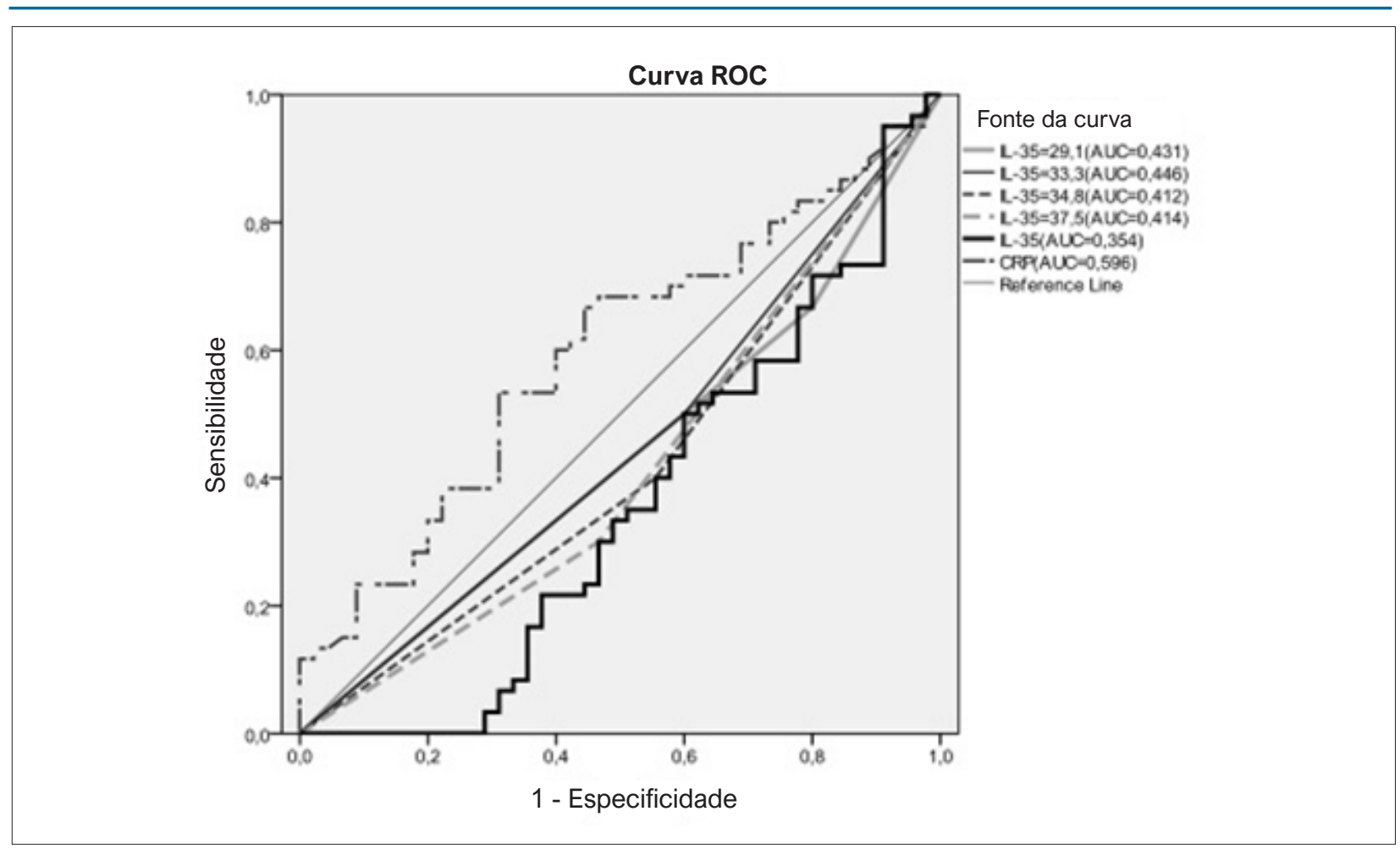

Figura 3 - Poder diagnóstico e pontos de corte de valores de IL-35 e PCR em relação à presença da DAC (análise ROC).

Tabela 8 - Valores da Área sob a Curva (AUC) para IL-35 e pontos de corte substitutos

\begin{tabular}{llllc}
\hline & AUC & SE & IC95\% & p \\
\hline IL-35 & 0,354 & 0,056 & $0,24-0,46$ & $\mathbf{0 , 0 1 1}$ \\
\hline Ponto de corte=29,1 & 0,431 & 0,056 & $0,32-0,54$ & 0,22 \\
\hline Ponto de corte=33,3 & 0,446 & 0,056 & $0,33-0,55$ & 0,33 \\
\hline Ponto de corte=34,8 & 0,412 & 0,056 & $0,3-0,52$ & 0,14 \\
\hline Ponto de corte=37,5 & 0,414 & 0,056 & $0,3-0,52$ & 0,11 \\
\hline
\end{tabular}

Como o teste PCR-as é um marcador inflamatório conhecido para aterosclerose, é considerado como preditor independente da DAC. ${ }^{23}$ Embora os níveis de PCR-as sejam mais altos no grupo de pacientes do que no controle, não foram estatisticamente significativos em nosso estudo. Além disso, não houve correlação entre a PCR-as e a IL-35. Este achado pode parecer incompatível com a literatura, porém, em nossa opinião, isso pode se dever ao fato de que a PCR-as está mais relacionada à instabilidade da placa e associada a eventos cardiovasculares, e não à extensão da aterosclerose. Assim, a IL-35 pode pode ser um marcador melhor do que a PCR-as para DAC estável.

Além disso, Wang et al., ${ }^{19}$ conduziram um estudo in vitro com animais e fez com que as células T regulatórias de ratos secretassem IL-35 para tratar a doença inflamatória intestinal e artrite induzida pelo colágeno, conhecidas por estarem relacionadas à inflamação crônica. ${ }^{19}$ Assim, a regulação celular da expressão de IL-35 pode ser um novo tratamento para DAC.

A principal limitação do nosso estudo foi seu desenho em centro único e o número relativamente baixo de participantes. Em segundo lugar, só avaliamos a DAC por meio da AGC; porém, outros métodos, como o ultrassom intravascular e a tomografia de coerência óptica, que são capazes de determinar a morfologia da placa, aumentariam o poder deste estudo. Por fim, o estudo seria mais completo se pudéssemos medir outras citocinas inflamatórias, como fator de crescimento tumoral beta e interleucina-10; porém, tínhamos um orçamento limitado.

\section{Conclusão}

A IL-35 é uma nova citocina que tem efeitos imunossupressores e anti-inflamatórios. ${ }^{24}$ Os principais achados deste estudo são os níveis baixos de IL-35 em pacientes com DAC estável, principalmente com GS alto, e a correlação negativa entre IL-35 e SS. Esses achados sugerem que os níveis baixos de IL-35 estão associados à extensão e à gravidade de DAC. Além disso, a secreção de IL-35 externamente induzida pode ser usada para o tratamento 
de aterosclerose. Mais estudos devem ser realizados em populações maiores.

\section{Contribuição dos autores}

Concepção e desenho da pesquisa: Oflar E, Sahin $M H$, Demir B, Ertugrul AS, Caglar FNT; Obtenção de dados: Oflar E; Análise e interpretação dos dados: Oflar E, Caglar FNT; Redação do manuscrito: Oflar E, Oztas DM, Beyaz MO, Ugurlucan M, Caglar FNT; Revisão crítica do manuscrito quanto ao conteúdo intelectual importante: Oflar E, Sahin MH, Demir B, Ertugrul AS, Oztas DM, Beyaz MO, Ugurlucan M, Caglar FNT.

\section{Potencial conflito de interesse}

Não há conflito com o presente artigo

\section{Referências}

1. White HD, Chew DP. Acute myocardial infarction. Lancet. 2008;372(9638):570-84.

2. Libby P. Current concepts of the pathogenesis of the acute coronary syndromes. Circulation 2001;104(3):365-72.

3. Lusis A J. Atherosclerosis. Nature. 2000;(6801):233-41.

4. Libby P. Molecular bases of the acute coronary syndromes. Circulation. 1995;91(11):2844-50.

5. Smith DA, Irving SD, Sheldon J, Cole D, Kaski JC. Serum levels of the antiinflammatory cytokine interleukin-10 are decreased in patients with unstable angina. Circulation. 2001;104(7):746-9.

6. Mazzone A, De Servi S, Vezzoli M, Fossati G, Mazzucchelli I, Gritti D, et al. Plasma levels of interleukin 2, 6, 10 and phenotypic characterization of circulating T lymphocytes in ischemic heart disease. Atherosclerosis. 1999;145(2):369-74

7. Anguera I, Miranda-Guardiola F, Bosch X, Filella X, Sitges M, Marin JL, et al. Elevation of serum levels of the anti-inflammatory cytokine interleukin-10 and decreased risk of coronary events in patients with unstable angina. Am Heart J. 2002;144(5):811-7.

8. Kilic T, Ural D, Ural E, YumukZ, Agacdiken A,Sahin T, et al. Relation between proinflammatory to anti-inflammatory cytokine ratios and long-term prognosis in patients with non-ST elevation acute coronary syndrome. Heart. 2006;92(8):1041-6.

9. Heeschen C, Dimmeler S, Hamm CW, Fichtlscherer S, Boersma E, Simmons $\mathrm{ML}$, et al. Serum level of the antiinflammatory cytokine interleukin-10 is an important prognostic determinant in patients with acute coronary syndromes. Circulation. 2003;107(16):2109-14.

10. Lebastchi AH, Qin L, Khan SF, Zhou J, Geirsson A, Kim RW, et al. Activation of human vascular cells decreases their expression of transforming growth factor-beta. Atherosclerosis. 2011;219(2):417-24

11. Ji QW, Guo M, Zheng JS, Mao XB, Peng YD, Li SN, et al. Downregulation of T helper cell type 3 in patients with acute coronary syndrome. Arch Med Res. 2009;40(4):285-93.

12. Niedbala W, Wei XQ, Cai B, Hueber AJ, Leung BP, et al. IL-35 is a novel cytokine with therapeutic effects against collagen-induced arthritis through the expansion of regulatory T cells and suppression of Th17 cells. Eur J Immunol. 2007;37(11):3021-9.

\section{Fontes de financiamento}

O presente estudo não teve fontes de financiamento externas.

\section{Vinculação acadêmica}

Este artigo é parte de tese de doutorado de Ersan Oflar pelo Bakirkoy Dr Sadi Konuk Training and Research Hospital.

\section{Aprovação ética e consentimento informado}

Este estudo foi aprovado pelo Comitê de Ética do Bakirkoy Dr Sadi Konuk Training and Research Hospital sob o número de protocolo 2014/03. Todos os procedimentos envolvidos nesse estudo estão de acordo com a Declaração de Helsinki de 1975, atualizada em 2013. O consentimento informado foi obtido de todos os participantes incluídos no estudo.

13. Collison LW, Workman CJ, Kuo TT, Boyd K, Wang Y, et al. The inhibitory cytokine IL-35 contributes to regulatory T-cell function. Nature. 2007;450(7169):566-9.

14. Devergne O, Hummel M, Koeppen H, Le Beau MM, Nathanson EC,KieffE, et al. A novel interleukin-12 p40-related protein induced by latent EpsteinBarr virus infection in B lymphocytes. J Virol. 1996;70(2):1143-5.

15. Devergne O, Birkenbach M, Kieff E. Epstein-Barr virus-induced gene 3 and the 35 subunit of interleukin 12 form a novel heterodimeric hematopoietin. Proc Natl Acad Sci. 1997;94(22):12041-6.

16. Gensini GG. A more meaningful scoring system for determining the severity of coronary heart disease. Am J Cardiol. 1983;51(3):606.

17. Serruys PW, Morice MC, Kappetein AP, Colombo A, Holmes DR, Mack MJ, et al. Percutaneous coronary intervention versus coronary-artery bypass grafting for severe coronary arterydisease. N Engl J Med. 2009;360(10):96172.

18. Lin Y, Huang Y,Lu Z, Luo C, Shi Y, Zeng K, et al. Decreased plasma IL-35 levels are related to the left ventricular ejection fraction in coronary artery diseases. PLoS One. 2012;7(12):e52490.

19. Wang B, Dai S The modulation of endoplasmic reticulum stress by chemical chaperone upregulates immune negative cytokine IL-35 in apolipoprotein E-deficient mice. PLoS One. 2014 Jan 30;9(1):e87787.

20. Bettini M, Castellaw AH, Lennon GP, Burton AR, Vignali DA (2012). Prevention of Autoimmune Diabetes by Ectopic Pancreatic-Cell Expression of Interleukin-35. Diabetes.2012;61(6):1519-26.

21. Kempe S, Heinz P, Kokai E, Devergne O, Marx N, Wirth T. Epstein-barr virus-induced gene- 3 is expressed in human atheroma plaques. Am J Pathol. $2009 ; 175(1): 440-7$.

22. Li Y, Wang Y, Liu Y, Wang Y, Zuo X, Lu X. The possible role of the novel cytokines il-35 and il-37 in inflammatory bowel disease. Mediators Inflamm. 2014;2014:136329.

23. Kuller LH, Tracy RP, Shaten J, et al. For the MRFIT Research Group: Relation of Creactive protein and coronary heart disease in the MRFIT nested casecontrol study. Am J Epidemiol. 1996;144(6):537-47.

24. Xinyuan L, Jietang M, Anthony V, Ying Y. IL-35 Is a Novel Responsive Antiinflammatory Cytokine - A New System of Categorizing Anti-inflammatory Cytokines. PLoS One. 2012;7(3):e33628. 